\title{
Determinación de las propiedades dielectricas de los combustibles, sus mezclas y del suelo, así como su impacto \\ en un uso eficiente de los recurso energéticos y en la determinación de la contaminación ambiental
}

\section{Determination of the dielectric properties of fuels, their mixtures and the soil. Their impact on the efficient use of energy resources and on the determination of environmental contamination}

\author{
DOI: https://doi.org/10.17981/ijmsor.04.01.04
}

Research Article - Reception Date: Apr 25, 2019- Acceptance Date: Jun 20, 2019

\author{
Francisco García Reina, Amarilys Méndez García, Lázaro Martínez Ibáñez \\ Universidad de Ciego de Ávila (Cuba) \\ pancho@ingenieria.unica.cu, amarilys@ingenieria.unica.cu, lazarom@ingenieria.unica.cu
}

To reference this paper:

F. García Reina, A. Méndez García, L. Martínez Ibáñez, "Determinación de las propiedades dielectricas de los combustibles, sus mezclas y del suelo, así como su impacto en un uso eficiente de los recurso energéticos y en la determinación de la contaminación ambiental”, IJMSOR, vol. 4, no. 1, 2019. https://doi. org/10.17981/ijmsor.04.01.04

Resumen-- El estudio de las propiedades dieléctricas de los materiales es una de las principales herramientas usadas actualmente para su caracterización, tanto estructuralmente como desde el punto de vista de las interacciones moleculares. En nuestra Universidad se ha venido utilizando en el estudio de los combustibles y sus mezclas: gasolina-alcohol y biodiesel-Diesel fósil, ya que permite determinar los mecanismos de interacción molecular presentes en las mezclas, tanto de sustancias polares como apolares, lo cual determina las concentraciones óptimas de los componentes en la mezcla, en cuanto a su estabilidad, energía libre, entropía, entalpía de la evaporación, así como la energía de cohesión y de los mecanismos de relajación dieléctrica. Estas propiedades de los combustibles son las que determinan, en primera instancia, la eficiencia de la combustión. Por otro lado, la misma técnica aplicada a los suelos permite determinar la humedad y lo que es más importante, la contaminación de los mismos por combustibles y lubricantes. Luego, con el mismo montaje experimental se pueden determinar las propiedades estructurales y dinámicas de los combustibles, sus mezclas y lubricantes, las cuales están estrechamente relacionadas con el uso eficiente de estos, así como la contaminación de los suelos por estos mismos agentes químicos. Se presentan los resultados de la caracterización dieléctrica y su relación con las energías de interacción molecular, de las mezclas alcohol-gasolina, biodiesel-Diesel fósil y la determinación de la influencia de la humedad y de la contaminación por combustibles en las propiedades dieléctricas de los suelos.

Palabras clave-- Propiedades dieléctricas; líquidos; interacción molecular; mezclas alcohol-gasolina; biodiesel; contaminación de los suelos
Abstract-- The study of the dielectric properties of materials is one of the main tools currently used for their characterization, both structurally and from the point of view of molecular interactions. At our University it has been used using the study of fuels and their mixtures: gasoline-alcohol and fossil biodiesel-Diesel, since it allows determining the molecular interaction mechanisms present in the mixtures, both of polar and non-polar substances, which determines the optimal dimensions of the components in the mixture, in terms of their stability, free energy, entropy, enthalpy of evaporation, as well as the energy of cohesion and dielectric relaxation mechanisms. These properties of fuels are what determine, in the first instance, the combustion efficiency. On the other hand, the same technique applied to soils makes it possible to determine the humidity and, more importantly, the contamination of the soils by fuels and lubricants. Then, with the same experimental setup, the structural and dynamic properties of fuels, their mixtures and lubricants can be determined, which are closely related to the efficient use of these, as well as the contamination of soils by these same chemical agents. The results of the dielectric characterization and its relationship with the molecular interaction energies of the alcohol-gasoline mixtures, biodiesel-fossil diesel and the determination of the influence of humidity and fuel contamination on the dielectric properties of the floors.

Keywords-- Dielectric properties; liquids; molecular interaction; alcohol-gasoline blends; biodiesel; Pollution of soils 


\section{INTRODUCCIÓN}

En el anterior Congreso Universidad 2010 los autores [1] presentaron un trabajo acerca de conjunto de tecnologías digitales de medición de alta precisión, usadas en las investigaciones de Eficiencia Energética llevadas a cabo en la Universidad, dirigidas a lograr un uso más racional y eficiente de la energía en la provincia y como apoyo fundamental a las tesis de Maestría, que, sobre este tema, se desarrolla en la Universidad desde el 2007, en convenio con la Universidad de Cienfuegos. Estas tecnologías de medición se pudieron montar gracias a los equipos de laboratorio de Física procedentes de la República Popular China, recientemente adquiridos por el Ministerio de Educación Superior y puestos a disposición de todas las Universidades del país. Si bien todas las tecnologías de medición puestas a punto han tenido una amplia aplicación en la práctica socioeconómica del territorio, es indudable que el mayor impacto se ha logrado con la medición de las propiedades dieléctricas de los materiales y combustibles, debido a su precisión, exactitud y a la gran cantidad de información que revela acerca de los mecanismos de interacción molecular y su influencia en las propiedades termodinámicas de las sustancias. La medición de las propiedades dieléctricas de los combustibles y sus mezclas permite determinar los mecanismos de interacción molecular presentes en las mezclas, tanto de sustancias polares como apolares, lo cual determina las concentraciones óptimas de los componentes en la mezcla, en cuanto a su estabilidad, energía libre, entropía, entalpía de la evaporación, así como la energía de cohesión y de los mecanismos de relajación dieléctrica. Estas propiedades de los combustibles son las que determinan, en primera instancia, la eficiencia de la combustión. Por estas razones, esta temática de investigación ha tenido una amplia difusión en todo el mundo contemporáneo y el hecho de tener estos equipos en el país, permite que nos podamos insertar de lleno en esta corriente principal de desarrollo científico-tecnológico [2], [3]. Por otro lado, la misma técnica aplicada a los suelos permite determinar la humedad y lo que es más importante, la contaminación de los mismos por combustibles y lubricantes, debido al hecho de que la constante dieléctrica del agua es mucho mayor que la media de los suelos y a su vez, la de los combustibles y lubricantes es mucho menor, por lo que el complejo formado por el suelo, el agua y los contaminantes tiene una respuesta dieléctrica fuertemente dependiente de las concentraciones de estos componentes en el suelo, de tal manera que pequeñas concentraciones de estos son fácil y exactamente determinados por el equipamiento. Por supuesto, esto también ha sido objeto de una gran cantidad de investigaciones recientes en todo el mundo y nos permite insertarnos de igual manera en esta corriente [4]. A continuación, se presentan los principales resultados obtenidos usando esta tecnología de caracterización dieléctrica de las sustancias en el estudio de las mezclas alcohol-gasolina, biodieselDiesel fósil y la determinación de la influencia de la humedad y de la contaminación por combustibles en las propiedades dieléctricas de los suelos.

\section{Desarrollo}

\section{A. TEORÍA}

El estudio de las propiedades dieléctricas de las sustancias es desde hace más de un siglo una poderosa herramienta para estudiar el comportamiento tanto estático como dinámico de los materiales. La medición de la constante dieléctrica compleja permite obtener información de los mecanismos de intercambio energético del campo eléctrico con las sustancias y por ende, da una valiosa información acerca de las propiedades de los combustibles, sus mezclas y el suelo, en este caso. Los metros LCR digitales permiten de forma rápida y segura medir impedancias y admitancias complejas, de tal modo que, con el software adecuado, se pueden determinar las partes reales e imaginarias de las capacitancias e inductancias de cualquier elemento de circuito. La permitividad dieléctrica relativa compleja se define como [2]:

$$
\varepsilon_{r}(\omega)=\varepsilon_{r}^{\prime}(\omega)+i \varepsilon_{r}^{\prime \prime}(\omega)
$$

Donde la parte real de la constante dieléctrica $\varepsilon_{\mathrm{r}}^{\prime}$ es una medida del intercambio conservativo de energía con el campo $\varepsilon$, mientras que la imaginaria $\mathrm{r}^{\prime \prime}$ lo es del intercambio disipativo, principalmente en forma de calor [4]:

$$
\begin{aligned}
& \varepsilon_{r}^{\prime}=C_{p} / C_{0} \\
& \varepsilon_{r}^{\prime \prime}=1 / \omega C_{0} R_{p}
\end{aligned}
$$

Donde:

$C_{\mathrm{p}}$ es la capacitancia equivalente en paralelo del capacitor con el medio líquido,

$\mathrm{C}_{0}$ es la capacitancia en vacío,

$R_{\mathrm{p}}$ es la resistencia equivalente en paralelo y

$\omega$ es la frecuencia angular.

Luego midiendo $(C, R)$ antes y después de llenar la celda con el líquido se obtiene la parte real y la imaginaria de la constante dieléctrica usando (2) y (3) [5], [2]. 
Con estos valores de $\varepsilon^{\prime}$ y $\mathcal{E}^{\prime \prime}, R_{\mathrm{p}}$ y $D$ es posible calcular todas las propiedades de interés de los combustibles y sus mezclas, así como del suelo. A continuación, se presentan las definiciones y expresiones matemáticas para el cálculo de estas propiedades en forma resumida y para más detalles ver la bibliografía citada.

La polarización molar $P$ es una medida de la respuesta de las moléculas del medio al campo eléctrico exterior, en función de sus momentos dipolares permanentes e inducidos y por tanto de la interacción dipolar de los enlaces que mantienen unidas a las moléculas en el líquido [6]:

$$
P=\left\{\frac{\left(\varepsilon_{m}-1\right)}{\left(\varepsilon_{m}+2\right)}\right\} V \quad P_{1}=\left\{\frac{\left(\varepsilon_{1}-1\right)}{\left(\varepsilon_{1}+2\right)}\right\} V_{1}
$$

Donde $P$ es la polarización molar de la mezcla y $P 1$ la del soluto, mientras que $\mathcal{E}_{\mathrm{m}}$ y $\mathcal{E}_{1}$ son las constantes dieléctricas de la mezcla y el soluto, y $V$ la fracción de volumen. La energía libre de Gibbs $\Delta G$ para la formación de la mezcla se calcula por [7]:

$$
\begin{aligned}
\Delta G & =-\frac{N}{2}\left[f_{2} \mu_{2}^{2}\left(R_{2}-R_{2}^{0}\right)+f_{2}^{2} \mu_{2}^{2}(g-1)\left(R_{2}-R_{2}^{0}\right)\right] \\
& =\Delta G_{0}+\Delta G_{\mathrm{rr}} .
\end{aligned}
$$

Donde $N$ es el número de moléculas polares por unidad de volumen, $\mathrm{f}_{1} \mathrm{y} \mathrm{f}_{2}$ son las fracciones molares de los dos componentes de la mezcla, $\mu_{2}$ el momento dipolar del soluto, $g$ el factor de correlación de Kirkwood que determina la interacción de las moléculas con sus vecinas más cercanas (corta distancia), $R_{2}^{0}$ y $R_{2}$ son los parámetros del campo de reacción de los líquidos puros y de la mezcla, respectivamente. El primer término $\Delta G_{0}$ da la energía de interacción dipolar a larga distancia y $\Delta G_{\mathrm{rr}}$ la de corta distancia y por tanto definen la estabilidad de la mezcla, así como la energía de cohesión que determina la entalpía de evaporación y por tanto la velocidad de combustión. El factor de Kirkwood se determina por [8]:

$$
\begin{aligned}
g \mu^{2}= & \frac{9 K T\left(2 \varepsilon_{m}+\varepsilon_{\infty}\right)^{2}}{4 \pi N f_{2}\left(\varepsilon_{\infty}+2\right)^{2}\left(2 \varepsilon_{m}+1\right)} \\
& \times\left[\frac{V\left(\varepsilon_{m}-1\right)}{\varepsilon_{m}}-\frac{3 V_{1} f_{1}\left(\varepsilon_{1}-1\right)}{2 \varepsilon_{m}+\varepsilon_{1}}-\frac{3 V_{2} f_{2}\left(\varepsilon_{\infty}-1\right)}{2 \varepsilon_{m}+\varepsilon_{\infty}}\right]
\end{aligned}
$$

Donde $K$ es la constante de Boltzmann, $T$ temperatura absoluta, $\varepsilon_{\infty}$ la constante dieléctrica óptica $\left(=n^{2}\right.$, $n$ es el índice de refracción). El factor de Bruggeman para la mezcla de dos líquidos A y B se define por [7]:

$$
\mathrm{f}_{\mathrm{B}}=\left(\frac{\varepsilon_{\mathrm{m}}-\varepsilon_{\mathrm{B}}}{\varepsilon_{\mathrm{A}}-\varepsilon_{\mathrm{B}}}\right)\left(\frac{\varepsilon_{\mathrm{A}}}{\varepsilon_{\mathrm{m}}}\right)^{1 / 3}=1-\mathrm{V}
$$

De su definición queda claro que $\mathrm{fB}$ debe de aumentar linealmente con la fracción de volumen del soluto, luego cualquier desviación de este comportamiento lineal es una clara indicación de una fuerte interacción dipolo-dipolo, que puede suministrar la energía libre necesaria para la ruptura de la mezcla. La tangente de pérdidas dieléctricas se define como el cociente entre la parte imaginaria y la real:

$$
\tan \delta=\varepsilon^{\prime} k^{\prime} \text {. }
$$

Usando la ecuación de Debye para las mezclas [8]:

$$
\tan \delta=\frac{\left(\varepsilon^{\prime}+2\right)^{2}}{\varepsilon^{\prime}} \cdot \frac{4 \pi \times \mathrm{N} \mu^{2}}{27 \mathrm{KT}} \cdot \frac{\omega \tau}{\left(1+\omega^{2} \tau^{2}\right)}
$$

Donde $\omega$ es la frecuencia angular de la señal y $\tau$ es el tiempo de relajación dieléctrica del mecanismo de interacción dipolar. Luego un gráfico de $\tan \delta$ vs. $\left(\varepsilon^{\prime}+2\right)^{2} / \mathcal{E}^{\prime}$ debe dar una recta, cuya pendiente permite calcular el valor de $\tau$, ya que todas las demás variables son conocidas.

En el caso de los suelos se tiene que la constante dieléctrica viene dada por:

$$
\varepsilon=\left[\theta \varepsilon_{w}^{\beta}+(1-\phi) \varepsilon_{s}^{\beta}+(\phi-\theta) \varepsilon_{\text {air }}^{\beta}\right]^{\frac{1}{\beta}}
$$

Donde $\theta$ es la concentración de agua libre en el suelo, $\varphi$ es la porosidad, $\beta$ es un parámetro referido a la geometría de los granos y su distribución espacial $(\approx 0,5), \varepsilon_{\mathrm{w}}, \varepsilon_{\mathrm{s}} \mathrm{y} \varepsilon_{\text {air }}$ son las constantes dieléctricas del agua, del suelo y del aire, respectivamente. Sin embargo, si hay contaminación por Diesel esta expresión se modifica a [10]:

$$
\varepsilon=\left[\theta \varepsilon_{w}^{\beta}+\psi \varepsilon_{\text {diesel }}^{\mathcal{\beta}}+(1-\phi) \varepsilon_{s}^{\beta}+(\phi-\theta-\psi) \varepsilon_{\text {air }}^{\mathcal{\beta}}\right]^{\frac{1}{\beta}}
$$

Donde $\psi$ es la fracción de volumen de Diesel y $\mathcal{E}_{\text {diesel }}$ es su constante dieléctrica. En esta expresión lo importante es que la constante dieléctrica del agua es del orden de 80, la del suelo seco es del orden de 20, mientras que la del Diesel es solo 3, por lo que pequeñas concentraciones de agua y Diesel en el suelo son fácilmente discriminadas en la medición [3].

\section{Materiales y MÉTodos}

El equipamiento utilizado y la metodología de la medición se describieron ampliamente en un trabajo anterior [1], donde los metros LCR XJ2811C (China) reúnen todos los requerimientos para el estudio tanto de dispositivos como materiales dieléctricos y magnéticos. Todas las mediciones se hicieron a una 
frecuencia de $10 \mathrm{kHz}$. En la Fig. 1 se presenta la foto del equipamiento utilizado para las mediciones:

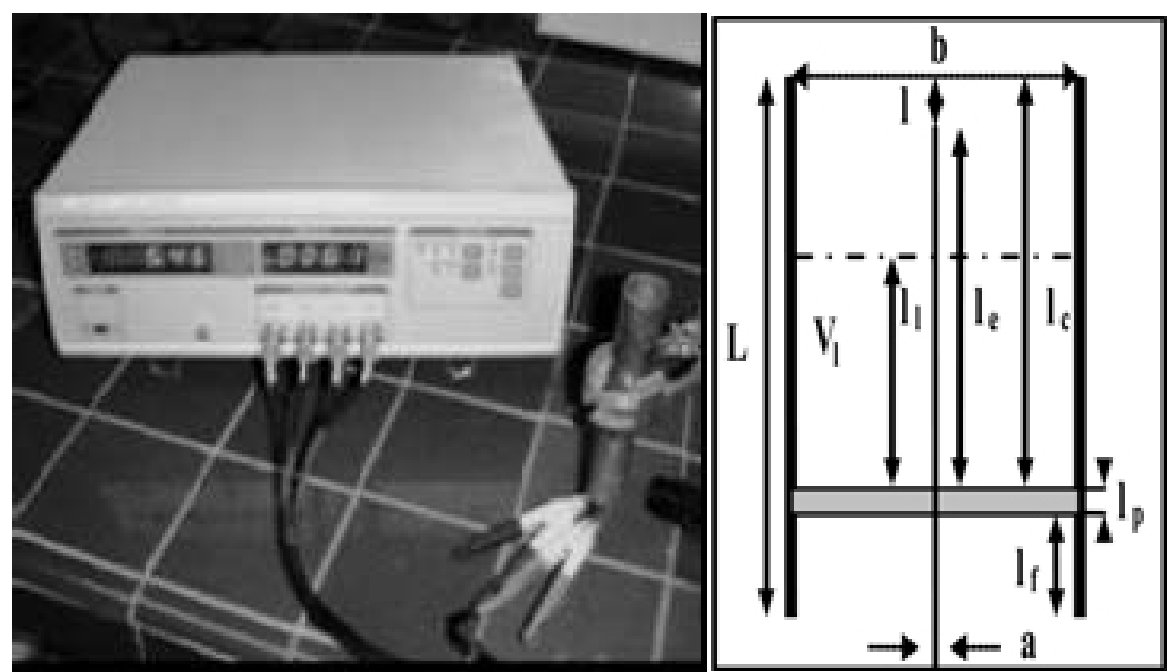

Fig. 1. Metro LCR digital y celda cilíndrica capacitiva, para las mediciones, a la derecha modelo de la celda cilíndrica.

Las mezclas de alcohol-gasolina se hicieron con los tres tipos de gasolina en uso en Cuba: regular, normal y especial, donde en este trabajo sólo se presentan los resultados de la regular, pero son típicos para los tres. Se usó alcohol etílico puro (grado espectroscópico) para las mezclas, las cuales se hicieron por fracción de volumen de alcohol, hasta un 30-35\%, debido a la separación espontánea de la mezcla a concentraciones mayores de alcohol [8]. El biodiesel se obtuvo por transestirificación del aceite de la Jatropha curcas, como se detalla en [10], sin procesamiento ulteriores de lavado, y el Diesel fósil se obtuvo de los laboratorios de combustible de la OBE en Ciego de Ávila. Las mezclas se efectuaron por fracción de Diesel fósil con el biodiesel desde 0 hasta el $100 \%$. Para el estudio en suelos se usó el suelo ferralítico rojo compactado (SFR), bajo pasto de la estación experimental del Centro de Bioplantas de la Universidad de Ciego de Ávila, al cual se le hicieron todos los análisis en el laboratorio de suelos de la UNICA. La muestra de suelo se tamizó a

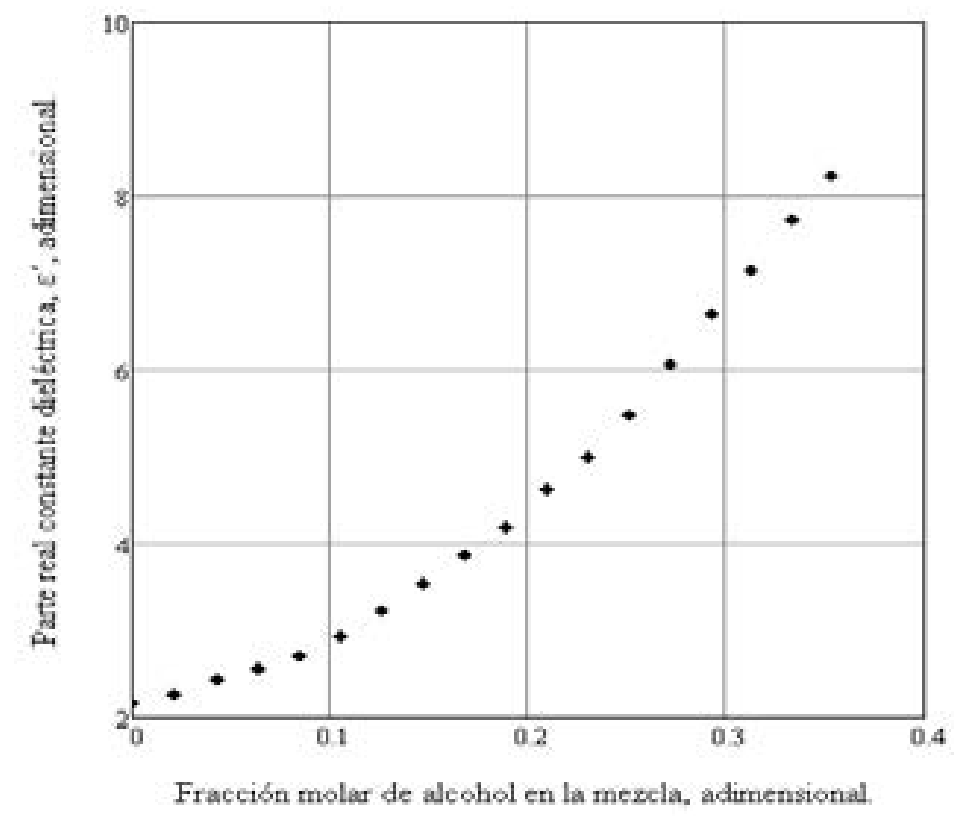

$1 \mathrm{~mm}$ y se secó en horno a $120^{\circ} \mathrm{C}$ por 2 horas. Se añadieron porciones en pesos de agua destilada y Diesel, para la preparación de las muestras y se usó el mismo capacitor cilíndrico que para los líquidos [3].

\section{Resultados}

\section{A. MEZCLA ALCOHOL-GASOLINA}

En la Fig. 2 se presentan la dependencia de la parte real y la imaginaria de la constante dieléctrica de la mezcla alcohol-gasolina. Si bien la gasolina es a su vez una mezcla, pero con una constante dieléctrica $\tan$ baja $(\approx 2)$, se puede afirma que todos su componentes son apolares y con muy baja polarizabilidad (benceno, naftalenos, parafinas, etc.), los cuales a los efectos de de la interacción con el campo eléctrico externo se comporta como un líquido apolar homogéneo, mientras que el alcohol, por el contrario, es una molécula altamente polar, con un alto momento dipolar permanente y alta polarizabilidad, por lo que su constante dieléctrica es $=24$. Es decir podemos estudiar la mezcla de alcohol gasolina con el mismo método que la de alcohol en benceno, por ejemplo [2].

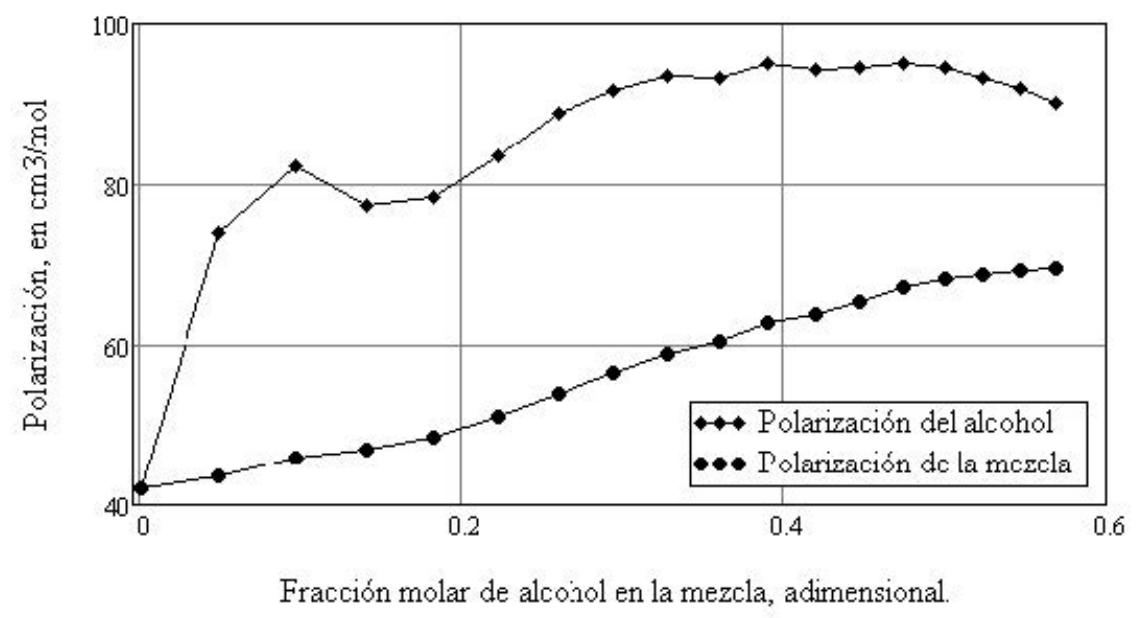

Fig. 3. Polarización molar de la mezcla y el alcohol en función de la fracción molar.

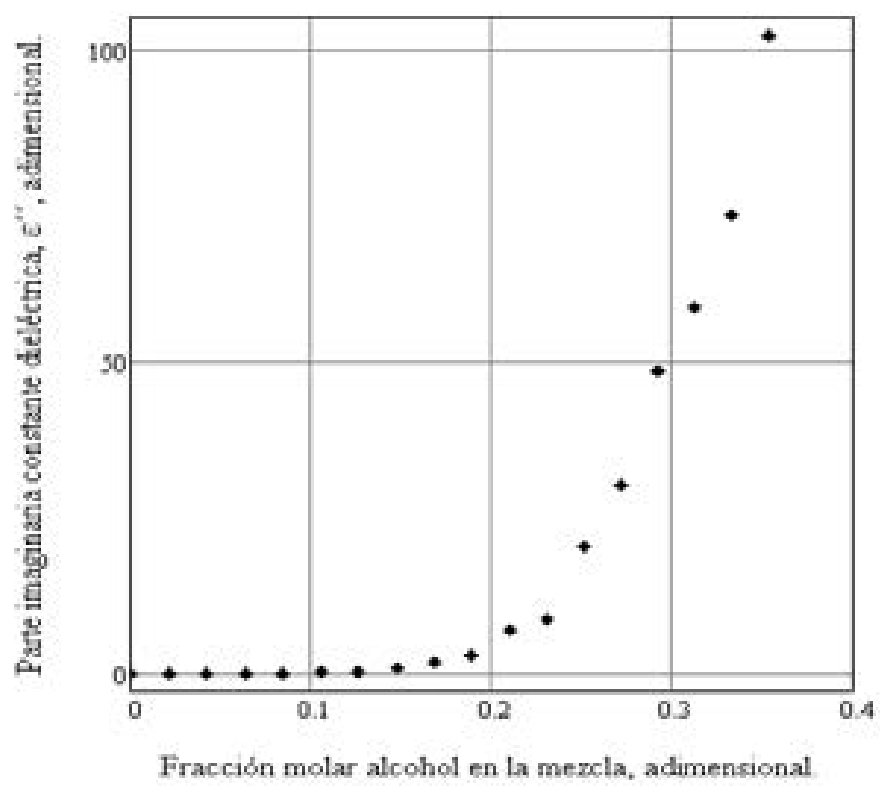

Fig. 2. Partes real e imaginaria de la mezcla alcohol gasolina en función de la fracción de alcohol. 
En la Fig. 3 se presenta las polarizaciones molares del alcohol y la mezcla calculadas con la expresión (4), donde queda claro que la interacción dipolo-dipolo de las moléculas de alcohol en la mezcla es la predominante en la energía de interacción molecular, lo que se pone de manifiesto en la Fig. 4 la cual da una clara imagen de los mecanismos involucrados en la interacción de las moléculas polares de alcohol con las apolares de la gasolina [6].

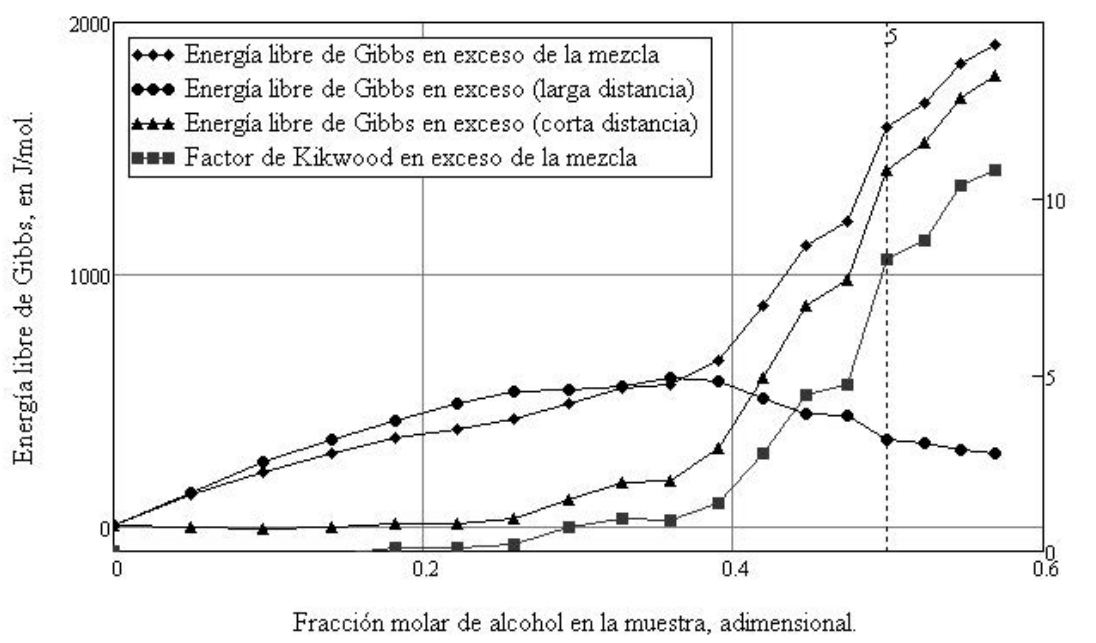

Fig. 4. Energía libre de Gibbs de la mezcla, a corta y larga distancia y factor Kirkwood.

Como se puede apreciar de esta figura, a bajas concentraciones de alcohol en la mezcla el mecanismo preponderante que aporta a la energía libre de Gibbs total $\Delta \mathrm{G}$ es la de la interacción a largas distancias
$\Delta G_{0}$, la cual llega a un máximo cerca del valor de 0,3 de fracción molar (0,18 de fracción de volumen), debido al creciente apantallamiento que provocan los dipolos inducidos en las moléculas apolares de los compuestos de la gasolina, los cuales a partir de esta concentración crecen rápidamente por la interacción a corta distancia $\Delta G_{\mathrm{rr}}$ y son los que dan la energía necesaria para la separación de la mezcla. El factor g de Kirkwood, que mide con precisión esta interacción a corta distancia tiene precisamente un comportamiento similar. Es de notar que a bajas concentraciones de alcohol $g<1$, lo que indica una alineación de los dipolos antiparalela al campo externo, debido a la interacción con los dipolos inducidos en las moléculas de gasolina [7].

En la Fig. 5 se presenta la dependencia del factor de Bruggeman con la fracción molar y el exceso de este factor respecto al comportamiento lineal.

De este gráfico es evidente a concentraciones en volumen hasta el $20 \%$ el mecanismo de interacción molecular hace que $\mathrm{f}_{\mathrm{B}}$ sea menor que el del comportamiento lineal y que por tanto a estas concentraciones la mezcla sea muy estable, sin embargo, a concentraciones mayores del $22 \%$, el mecanismo se invierte por completo y la mezcla es cada más inestable hasta que acaba por separarse cerca del $30 \%$ de alcohol. De hecho, todo este comportamiento debe de reflejarse en las pérdidas dieléctricas, como se muestra en la Fig. 6 [6], [5]:

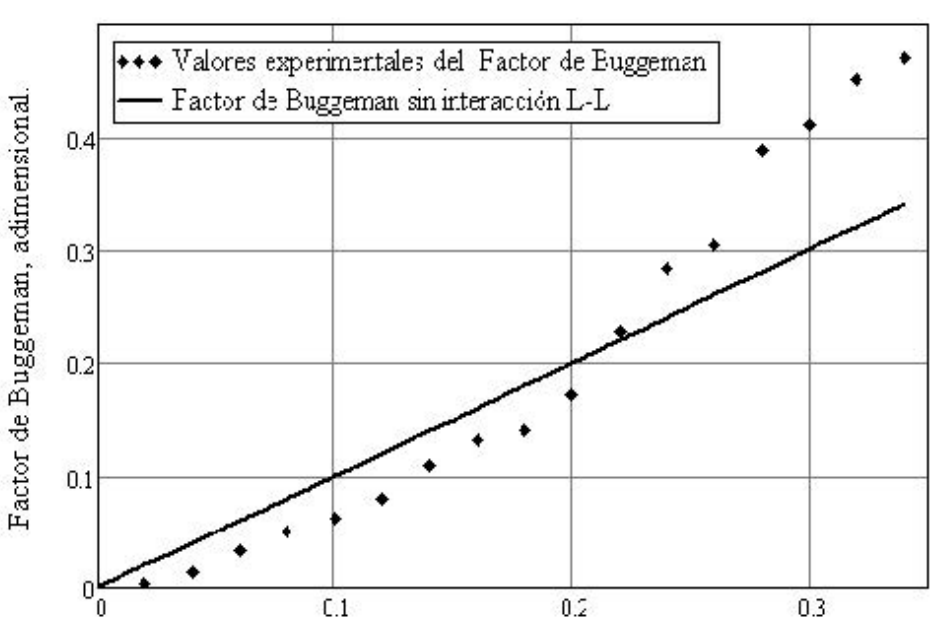

Fracción de volumen de alcohcl en la muestra, adimensicnal.

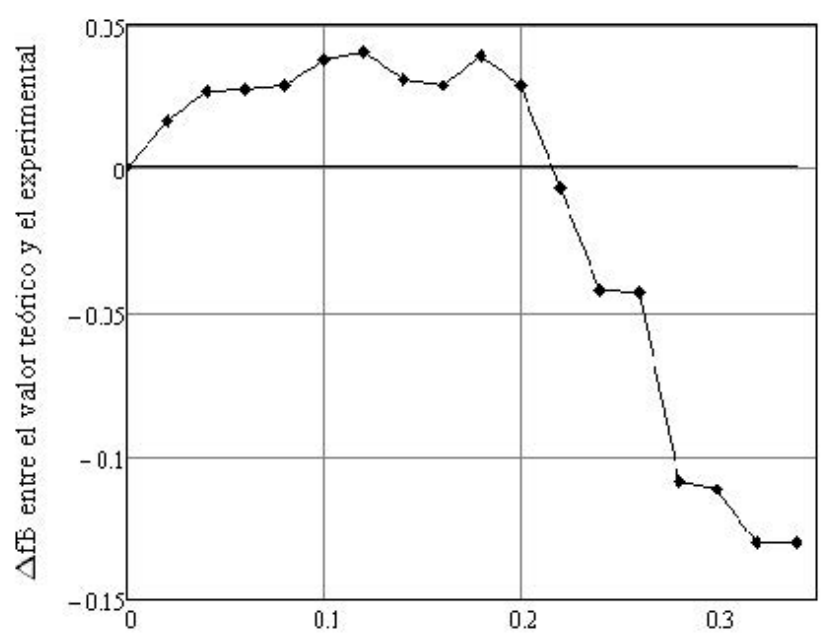

تracción de walumen de alcohol en la muesra, adimensional.

Fig. 5. Factor de Bruggeman $\mathrm{fB}$ y el exceso $\Delta \mathrm{fB}$ en función de la fracción de volumen.
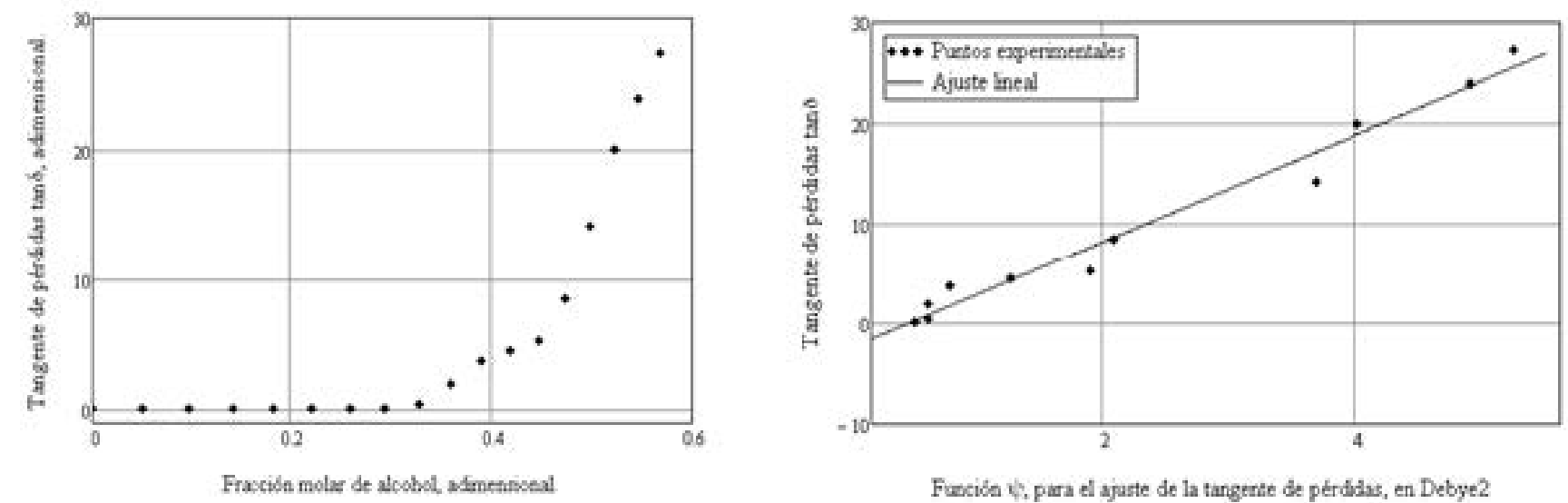

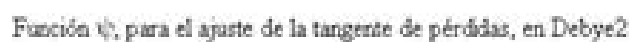

Fig. 6. Tangente de pérdidas y ajuste para el cálculo del tiempo de relajación $\tau$. 
Es evidente que el mecanismo de relajación presente de interacción dipolo-dipolo provoca un aumento de las pérdidas dieléctricas y el ajuste lineal según la ecuación (9) para el tiempo de relajación da el valor $\tau=(1,7$ $\pm 0,6) \cdot 10^{-7} \mathrm{~s}$, típico para este proceso de nucleación que trae por consecuencia la separación de los dos líquidos.

Todos estos resultados refuerzan la idea propuesta de usar las mezclas alcohol-gasolina hasta un $20 \%$ en volumen [8], que es donde la mezcla es más estable y disminuye las energías de interacción molecular que provocan una disminución de la entalpía de evaporación y por tanto aumentan la eficiencia de la combustión. También se demuestra que los enlaces formados por la interacción dipolar de las moléculas de alcohol con la de los compuestos de la gasolina, hacen que sus propiedades antidetonantes se refuercen [8], [9]. Luego, esta medición es de inestimable valor a la hora de evaluar la termodinámica de las mezclas alcohol gasolina.

\section{B. MEZCLA BIODIESEL-DIESEL FOSIL}

En la Fig. 7 se presenta las dependencias de las partes reales e imaginarias de las mezclas biodiesel-Diesel fósil, en función de la fracción de volumen de Diesel fósil.

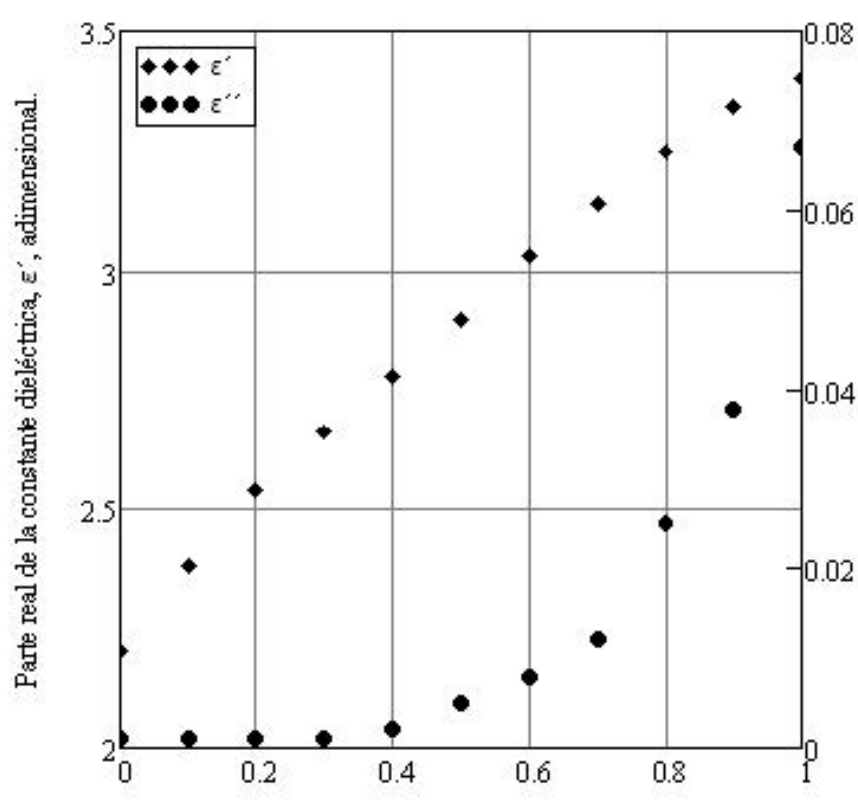

Fracción de volumen de Diesel en Biodiesel, adimensional
En este caso, ambos líquidos son apolares con muy baja constante dieléctrica por lo que los mecanismos vistos en el caso anterior no se presentan en esta mezcla. La $\varepsilon^{\prime}$ del biodiesel es casi el doble que la del Diesel fósil, debido a la alta concentración de esteres que contiene. La $\mathcal{E}^{\prime \prime}$ es también mayor en el biodiesel, por la misma razón, aunque no lo suficientemente alta para provocar mecanismos de relajación dieléctrica diferentes. Sin embargo, esta alta concentración de esteres en el biodiesel hacen que la energía liberada en la combustión sea menor que en el Diesel y los contaminantes en los gases de escape se incrementen, por lo que en el proceso de lavado de este deben de eliminarse estos esteres, y estas mediciones pueden probar la efectividad de este lavado. La dependencia de $\mathcal{E}^{\prime}$ con la fracción de volumen no es exactamente lineal, como han reportado otros autores [10], pero realmente no se desvía mucho de la linealidad, como se puede apreciar en la Fig. 8. Todo esto hace que esta mezcla sea absolutamente miscible en todo el rango de fracción de volumen, como se ve en la siguiente figura, donde el exceso factor de Bruggeman sólo cambia de signo a concentraciones de Diesel superiores al $80 \%$, y no se libera la energía suficiente para la separación de las mezclas [10].

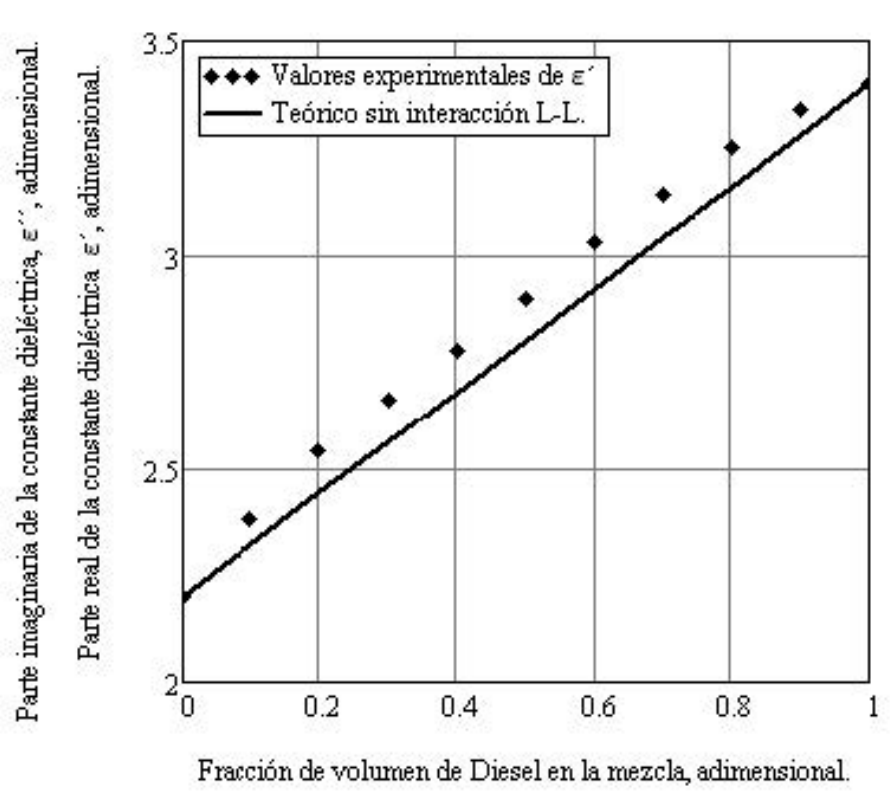

Fig. 7. Partes real e imaginaria de $\varepsilon$ de la mezcla biodiesel-Diesel en función de la fracción de Diesel fósil.

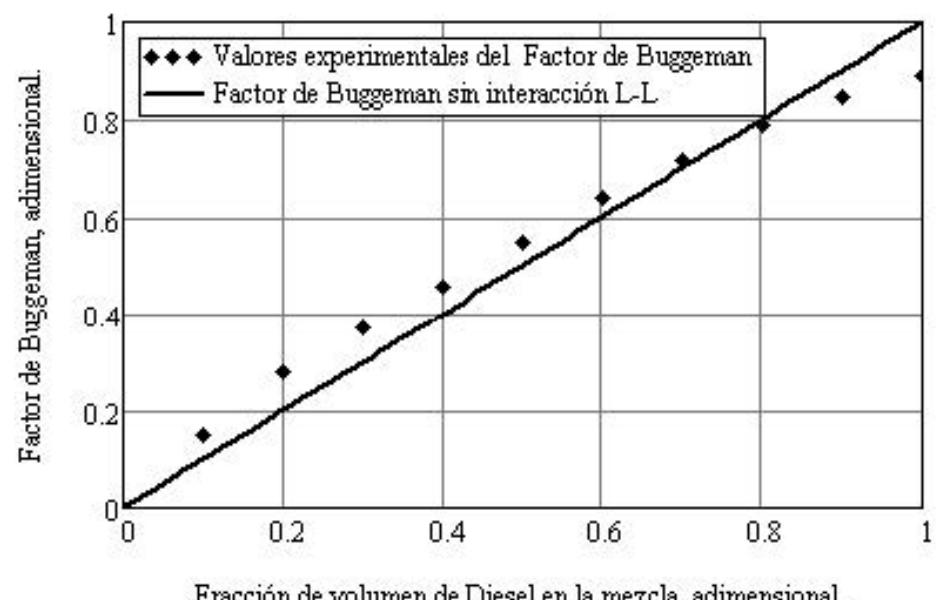

Fracción de volumen de Diesel en la mezcla, adimensional.

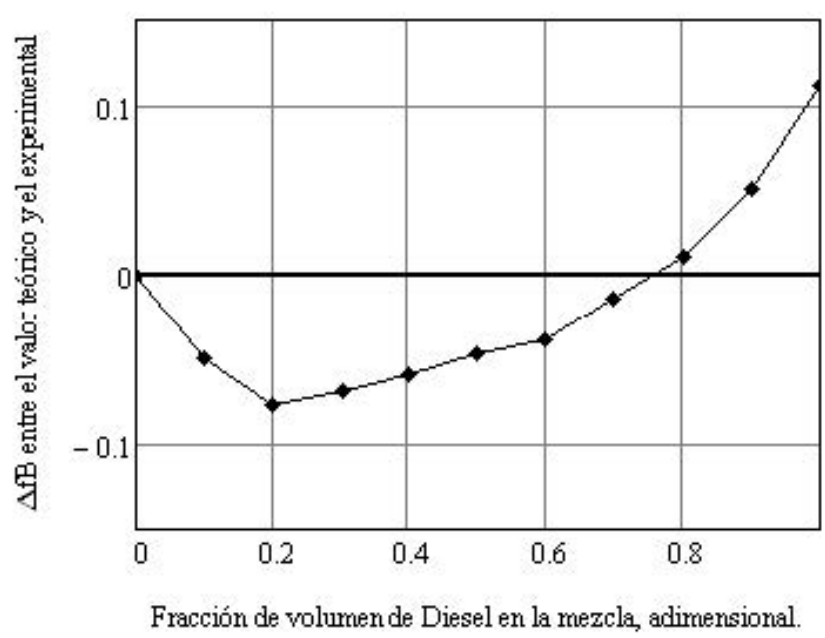

Fracción de volumen de Diesel en la mezcla, adimensional.

Fig. 8. Factor de Bruggeman $\mathrm{fB}$ y el exceso $\Delta \mathrm{fB}$ en función de la fracción de volumen. 


\section{SUELOS}

En la Fig. 9 se presenta la dependencia de la $\varepsilon^{\prime}$ del suelo para diferentes fracciones de volumen de agua y diferentes fracciones de volumen de Diesel. Los puntos son los valores experimentales y las curvas son los ajustes con las expresiones (10), para 0 fracción de volumen de Diesel, y (11) para las demás [12].

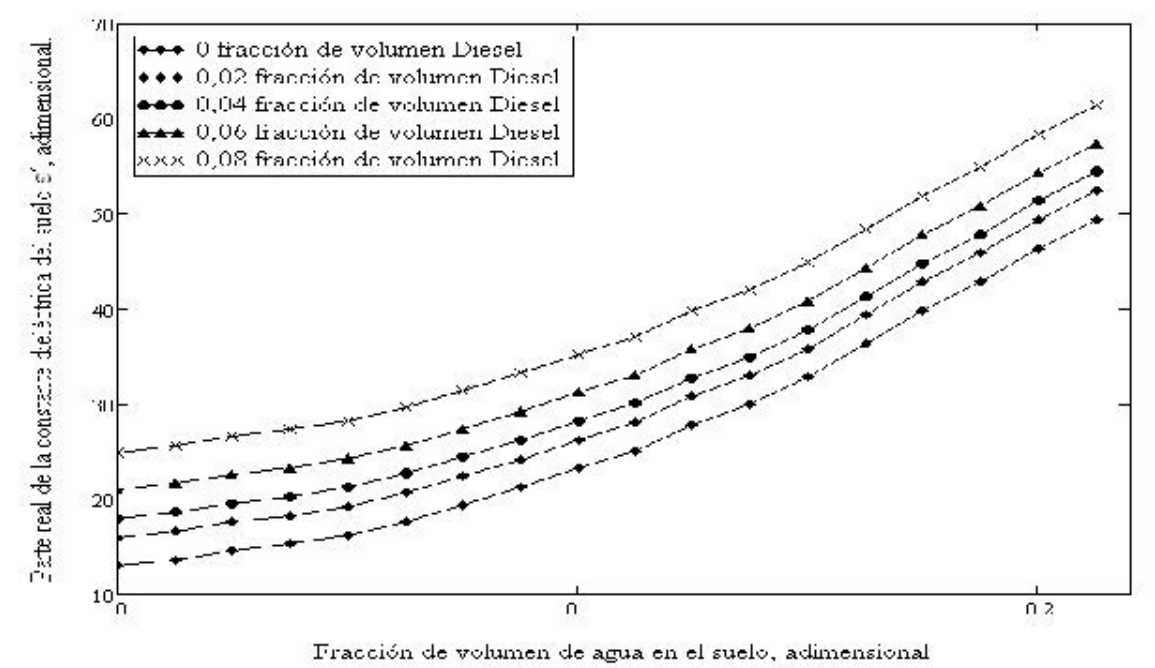

Fig. 9. $\varepsilon^{\prime}$ del suelo en función de las fracciones de volumen de agua y Diesel.

Estos resultados muestran que la respuesta del equipo es lo suficiente precisa para discriminar las concentraciones de agua y Diesel usadas en el experimento, por lo cual puede emplearse como sensor para determinar la humedad y la contaminación por Diesel en los suelos. En estos momentos se está montando un sistema de riego por aspersión en una casa de cultivo en la estación experimental de la Universidad, con un sistema automatizado en el que se usa este equipamiento como sensor de la humedad del suelo. Además, se están elaborando las curvas de calibración de humedad y concentración de Diesel para los diferentes tipos de suelo de la provincia.

\section{Conclusiones}

Los resultados alcanzados en este trabajo demuestran el gran valor práctico que tiene el estudio de las propiedades dieléctricas de los combustibles, sus mezclas y en la contaminación de los suelos, lo cual es posible con el equipamiento chino adquirido por el MES. En el caso de las mezclas alcohol gasolina se determinó las concentraciones máximas de alcohol permisible y las energías de interacción moleculares asociadas a los mecanismos de relajación dieléctrica presentes, en estas mezclas de un líquido polar en uno apolar, lo que permite evaluar la estabilidad de las mezclas y las energías de cohesión, que determinan finalmente la eficiencia de la combustión. En las mezclas biodiesel-Diesel fósil se determinó el aumento de la constante dieléctrica debido a la presencia de esteres, los cuales, dado su efecto nocivo en la combustión, deben ser eliminados por lavado y esta técnica puede medir este efecto. Por último, los resultados en los suelos demuestran la eficacia del método en la determinación de la humedad y la contaminación por Diesel, lo cual es de una gran aplicabilidad, teniendo en cuenta que los métodos tradicionales para esto son muy engorrosos y caros.

\section{REFERENCIAS}

[1] F. García y A. Martínez, "Uso de los laboratorios de Física de la UNICA para el desarrollo de tecnologías de medición de propiedades termodinámicas y su impacto en la evaluación de la eficiencia energética", ponencia presentada en el 7 Congreso Internacional de Educación Superior, Universidad 2010, La Habana, Cuba, Feb. 8-12, 2010.

[2] J. Duarte, W. Guillín y J. Sánchez, "Desarrollo de una metodología para la predicción del volumen real en la cámara de combustión de motores diésel utilizando elementos finitos", INGE CUC, 14(1), 122-132, 2008. https://doi.org/10.17981/ ingecuc.14.1.2018.11

[3] Rodríguez, L., Castellano, M., \& Caridad, M. (2017). Planificación estratégica de recursos humanos en empresas de consumo masivo. IJMSOR: International Journal of Management Science \& Operation Research, 2(1), 38-43. Recuperado de http://ijmsoridi.com/index.php/ijmsor/article/ view/84

[4] Y. Son, M. Oh and S. Lee. "Influence of diesel fuel contamination on the electrical properties of unsaturated soil at a low frequency range of $100 \mathrm{~Hz}-10 \mathrm{MHz}, J$ Environ Geol, vol. 58, no. 6, pp. 1341-1348, Jan. 2009. https://doi. org/10.1007/s00254-008-1637-x

[5] Agilent Solutions for Measuring Permittivity and Permeability with LCR Meters and Impedance Analyzers, AN 1369-1, Agilent Technologies, New York, USA, 2006.

[6] K. K. Gupta and P. J. Singh, "Fluid structure and molecular interactions of actophenone derivatives", Pramana J. of Physc., vol. 62, pp. 1129-1137, May. 2004. https://doi. org/10.1007/BF02705259

[7] S. R. Kumar and S. R. Sankar, "Dielectrics studies of binary mixture of polar liquids in non polar aolvents", J. In. Inst. Sci., vol. 72, no. 6, pp. 487-493, Dec. 1992. Available from http://journal.library.iisc.ernet.in/index.php/iisc/article/view/484

[8] P. Singh and K. S. Sharma, "Dielectric behaviour of ketoneamine binary mixtures at microwave frequencies", J. In. Inst. Sci., vol. 75, no. 4, pp. 259-274. Apr. 1996. Available from https://www.ias.ac.in/article/fulltext/pram/046/04/0259. 0270

[9] R. M Tshiteya, E. N. Vermiglio and J. W. Onstad, "The Impact of Phase Separation in Alcohol/gasoline Blends on the Existing Fuel Distribution System," in Proc. EC Conference, Reno, NV, USA, Aug. 1990. pp. 343-348. https://doi. org/10.1109/IECEC.1990.716513

[10] García-León, R., Echavez Díaz, R., \& Flórez Solano, E. (2018). Análisis termodinámico de un disco de freno automotriz con pilares de ventilación tipo NACA 66-209. INGE CUC, 14(2), 9-18. https://doi.org/10.17981/ingecuc.14.2.2018.01

[11] P. A. Sorichetti and S. D. Romano, "Physico-chemical and electrical properties for the production and characterization of Biodiesel", Phys Chem Liq, vol. 43, no. 1, pp. 37-48, Aug, 2006. https://doi.org/10.1080/0031910042000303536

[12] D. Chenaf, N, Amara and M. Tetreault, "Laboratory Investigation of the Effect of Diesel Contamination on the Unfrozen Water Content Curve of a Frozen Soil”, in Proc. TDR 2006, 3rd International Symposium and Workshop on Time Domain Reflectrometry for Innovative Soil Application, PU, W Lafayette, IN, USA, Sep. 17-20, 2008, Paper ID 47. 